\title{
Transarterial Chemoembolization for Hepatocellular Carcinoma
}

\author{
Thuong G. Van Ha, M.D. ${ }^{1}$
}

Hepatocellular carcinoma (HCC) is the fifth most common malignancy in the world and is responsible for $\sim 500,000$ deaths worldwide annually. ${ }^{1}$ The incidence almost equals the mortality. It is the most common cause of death in patients with compensated liver cirrhosis. HCC usually arises in the setting of chronic liver disease, commonly from hepatitis $\mathrm{B}, \mathrm{C}$, or alcohol abuse. In fact, $80 \%$ of $\mathrm{HCC}$ arises in the setting of cirrhosis. Despite available treatment, survival rates of HCC remain poor, with an estimation of $54 \%$ at 1 year, $40 \%$ at 2 years, and $28 \%$ at 3 years. $^{2}$ The only definitive therapy is liver transplantation. Resection, when possible, is not curative as cirrhosis remains a comorbidity. Even with transplantation in early HCC associated with cirrhosis, there is still a potential risk for $\mathrm{HCC}$ recurrence. ${ }^{3}$ Typically, transplantation is reserved for candidates with a single lesion up to $5 \mathrm{~cm}$ in diameter, or multiple lesions up to three with a total diameter of $9 \mathrm{~cm}$, with no vascular invasion and no regional or distant nodal metastatic disease. The 4-year survival rate for transplantation is $\sim 80 \%$. Similar survival rates have been reported for larger HCCs. ${ }^{4}$

With transplantation the only curative option, qualified patients often languish on the waiting list due to the current organ shortage. It has been shown that a 6- to 12-month waiting time for orthotopic liver transplantation (OLT) is associated with reduced overall survival compared with subjects with lesser waiting time due to progression of tumor beyond favorable criteria or due to drop-out for other reasons. ${ }^{5}$ In fact, the average waiting time can exceed one-year, at which point the drop-out rate is 30 to $40 \%$. Drop-out rate from HCC progression beyond favorable criteria is estimated at $10 \%$ at 6 months. ${ }^{6}$ Surgical resection can increase the OLT rate to $3.7 \%$ and $10.7 \%$ for a waiting list of 6 and 24 months, respectively. However, surgical resection is associated with significant risks in the setting of cirrhosis, including hemorrhage and hepatic decompensation. ${ }^{7}$ It is in this setting that other therapies have been developed to treat, though not necessarily cure, patients with $\mathrm{HCC}$ to prevent HCC progression and increase the chance of organ transplantation, to downstage patients into favorable criteria for liver transplantation, and to prolong survival and improve quality of life for those who do not qualify for liver transplantation. Therapies that have been in use include transarterial chemoembolization (TACE), ablative therapies (including both thermal and cryoablation), radio embolization, and percutaneous alcohol injection. The focus of this article is on the rationale, technique, and results of TACE in $\mathrm{HCC}$ in the setting of cirrhosis.

\section{TRANSARTERIAL CHEMOEMBOLIZATION}

\section{Rationale}

TACE is a percutaneous technique, usually through the common femoral arterial approach, making use of fluoroscopic guidance and coaxial catheter system to deliver a local and concentrated dose of chemotherapeutic agents directly into the arterial feeding vessels of the tumor in conjunction with or followed by embolization using either permanent or temporary particulate materials. Though techniques vary from institution to institution, the principles of TACE are consistent: to deliver a higher drug concentration into the tumor than possible by systemic therapy and prolonging drug dwell time within tumor by reducing washout. The significance of other effects including ischemia caused by embolization having a synergistic effect with the chemotherapeutic agents has not gained general acceptance. As most of the drug is retained within the tumor, another benefit is the reduction of systemic drug toxicity. Of note, TACE exploits the predominant hepatic arterial supply to

\footnotetext{
${ }^{1}$ Department of Radiology, University of Chicago Medical Center, Chicago, Illinois.

Address for correspondence and reprint requests: Thuong G. Van Ha, M.D., Department of Radiology, University of Chicago Medical Center, 5841 S. Maryland Avenue, MC 2026, Chicago, IL 60637

Visceral Arterial Intervention; Guest Editor, Sanjeeva P. Kalva, M.D. Semin Intervent Radiol 2009;26:270-275. Copyright (C) 2009 by Thieme Medical Publishers, Inc., 333 Seventh Avenue, New York, NY 10001, USA. Tel: +1(212) 584-4662.

DOI 10.1055/s-0029-1225672. ISSN 0739-9529. 
HCC, with occasional parasitic siphoning from other arterial territories depending on tumor location such as the intercostal, lumbar, inferior phrenic, or internal mammary arteries. The normal liver parenchyma is supplied mostly by the portal vein and a much smaller percentage by the hepatic artery.

\section{Goals}

Implicit in the statement that transplantation is the only cure for $\mathrm{HCC}$ in the setting of liver cirrhosis is that TACE is only a palliative procedure, albeit one that has demonstrated increased quality of life and prolonged survival. It is important, therefore, to educate patients to bring their expectations in line with what is known: TACE is not a curative procedure. $\mathrm{HCC}$ is a chronic disease; multiple procedures might be needed as long as there is a demonstrable objective benefit.

\section{PREPROCEDURE}

Much has been written about patient selection and detailed coverage of this area is beyond the scope of this article. However, certain aspects of patient selection must be recognized, including Child-Pugh classification, extrahepatic involvement, liver tumor burden, serology values, and portal vein patency. $\mathrm{Pa}$ tients with extrahepatic metastasis may be candidates if hepatic tumor burden is predominant and is the main cause of symptoms. Caution should be exercised in Child-Pugh class C patients as TACE is poorly tolerated in this group who are prone to hepatic failure. In patients with large hepatic tumor burden, the staging of treatment into two or more sessions might be necessary to avoid hepatic failure. In general, no more than $50 \%$ of liver volume should be chemoembolized at a time. Many physicians will not treat tumors that are bigger than $50 \%$ of total liver volume. Staged procedures are usually performed at least 2 weeks apart to allow patients to recover. More time may be needed in some patients to return to baseline. In patients with elevated liver enzymes in addition to increased bilirubin levels, there is a propensity for hepatic failure and TACE may be contraindicated. Portal vein thrombosis is not a contraindication if there is adequate collateral flow. However, in patients with portal vein thrombosis, it is judicious to do superselective chemoembolization to spare as much normal liver parenchyma as possible.

Cross-sectional imaging is mandatory prior to TACE to localize liver tumors, to assess portal vein patency, to look for other comorbid conditions such as bile duct obstruction (which must be decompressed prior to procedure), and to examine arterial anatomy for treatment planning.

\section{PERIPROCEDURE}

Hydration: Intravenous (IV) fluids such as normal saline $250 \mathrm{cc} /$ hour $\times 4$ hours then $150 \mathrm{cc} /$ hour $\times 6$ hours

Medications:

Antibiotics: Cefoxitin $2 \mathrm{~g}$ IV. If there is a history of biliary surgery, a more aggressive and longer course of antibiotics (10 days) is needed.

Other medications: Zofran ${ }^{\circledR} \quad$ (GlaxoSmithKline, Brentford, London, UK) $10 \mathrm{mg}$ IV push (IVP) or Reglan ${ }^{\circledR}$ (Baxter Healthcare, Deerfield IL) $1 \mathrm{mg}$ IVP, Decadron ${ }^{\circledR}$ (Merck \& Co., Inc., Whitehouse Station, NJ) $10 \mathrm{mg}$ IV

Foley catheter to gravity

Sedation with Fentanyl ${ }^{\circledR}$ (Janssen Pharmaceutica, Beerse, Belgium) and Versed ${ }^{\circledR}$ (Hoffman LaRoche, Nutley, NJ)

\section{PROCEDURE}

\section{Preliminary Angiography}

At the time of procedure, a routine abdominal aortogram is performed (Fig. 1). This delineates visceral anatomy and can identify tumor parasitization from intercostal, phrenic, or lumbar arteries. The superior mesenteric artery (SMA) is then selected with a VS-1 or RC-1 catheter (Boston Scientific, Natick, MA) and an arteriogram is performed with large contrast volume (e.g., $5 \mathrm{~mL}$ per second for $25 \mathrm{~mL}$ total volume) to assess for variant anatomy, unconventional feeding arteries to tumors, and in the venous phase, to assess for portal vein patency and flow. Next, the celiac artery is selected and a selective arteriogram is performed to look for arterial supply to the tumors and also to identify vessels that should not be embolized such as vessels to the gallbladder, stomach, and intestines (Fig. 2). Vessels to the stomach or intestines should not be chemoembolized and may rarely need to be coil embolized prior to chemoembolization if they are at risk for reflux. Once the arterial feeding vessels to the tumor(s) are identified, they are superselected for chemoembolization. Usually, due to small size of the tumor vessels a microcatheter, such as the Renegade Hi Flow ${ }^{\circledR}$ catheter (Boston Scientific, Natick, $\mathrm{MA}$ ), is used in a coaxial fashion. If super-selection is possible, the vessels are chemoembolized to stasis. Chemoembolization can be performed with ethiodol mixed with chemotherapeutic agents followed by embolization, with Gelfoam ${ }^{\mathbb{R}}$ (Pfizer Pharmaceuticals, New York, NY) or polyvinyl alcohol (PVA) particles (Boston Scientific, Natick, MA). Alternatively, TACE could be performed using drug-eluting beads. Super-selection is associated with better results in some studies. ${ }^{8}$ If there is more than one feeding vessel, the dose is divided among feeder vessels using subjective approximation to the volume of tumor fed by each vessel during arteriogram. 

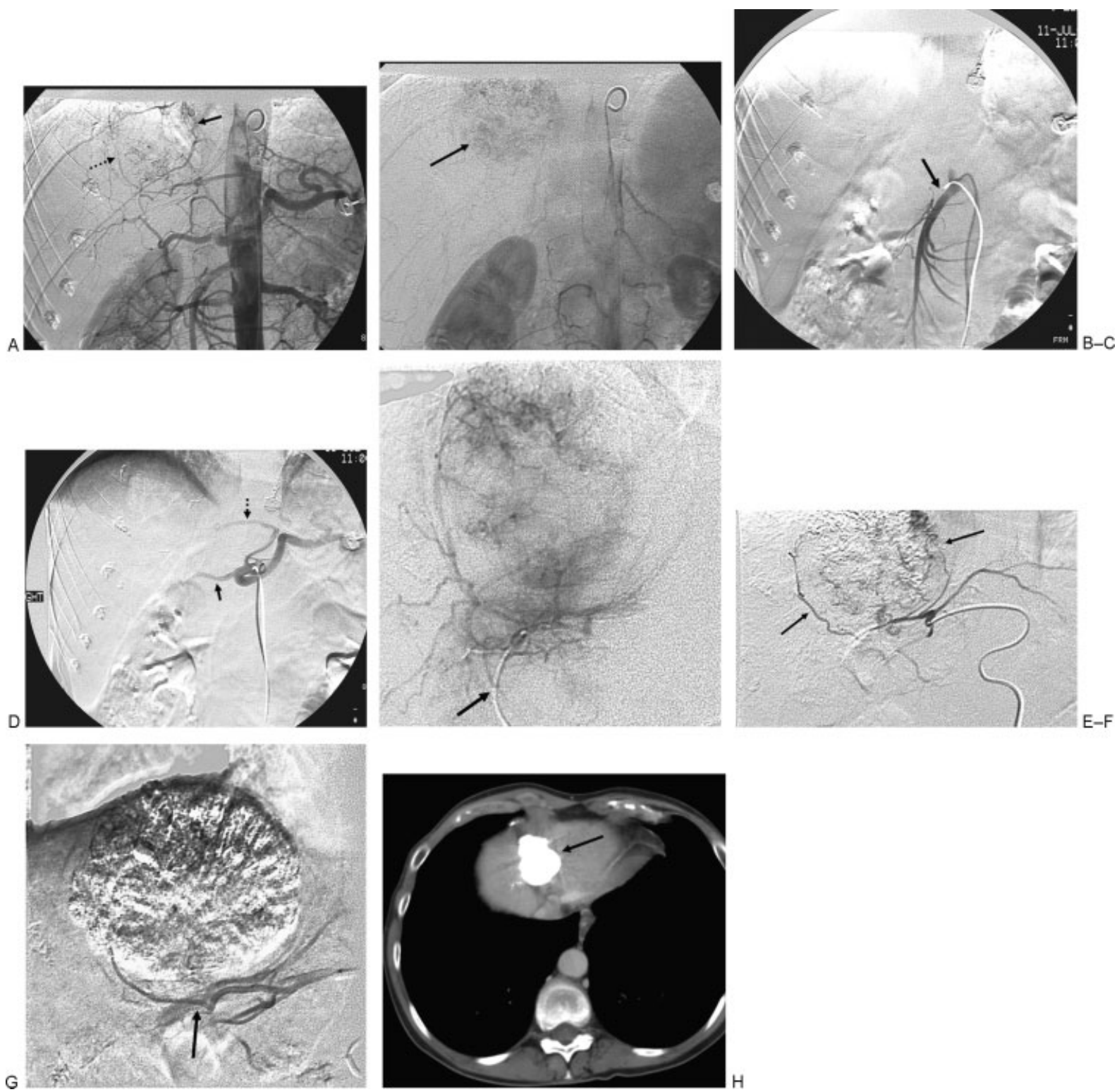

Figure 1 A 62-year-old man with cirrhosis and hepatocellular carcinoma (HCC) presents for transarterial chemoembolization (TACE) (A) Abdominal aortogram, late arterial phase, shows arterial supply from the right (dashed arrow) and left (arrow) hepatic arteries. (B) Abdominal aortogram, late venous phase, shows persistent blushing in the tumor (arrow). (C) Superior mesenteric arteriogram (arrow) shows normal anatomy (i.e., no replaced or accessory right hepatic artery or other variant). In the portal venous phase (not shown), the portal vein was patent. (D) Celiac arteriogram shows patent common hepatic artery (arrow) and a replaced left hepatic artery (dashed arrow). (E) Super-selective arteriogram of the feeding artery the right hepatic artery with a microcatheter (arrow) was performed followed by TACE. (F) Replaced left hepatic arteriogram shows feeders (arrows) to the tumor. (G) Postembolization angiogram of the feeders showed stasis (arrow) of the vessels and iodized oil staining of the tumor. (H) Follow-up computed tomography (CT) one year later showed residual iodized oil (arrow) within the tumor and no enhancement to suggest residual tumor.

The same is used when there are multiple lesions. Unless tumor burden is large, there is usually enough chemoagents to achieve near stasis or decreased flow, and stasis is achieved with Gelfoam ${ }^{\circledR}$ or particles. When using drug-eluting beads, larger tumor burden usually requires two vials of the beads instead of the usual one (see below).
In cases of larger tumors or tumors that are subcapsular, nonhepatic parasitic feeders should be considered especially if hepatic feeding vessels show an incomplete spherical blush. Dome lesions can receive supply from the right inferior phrenic artery. Anterior dome tumors can derive supply from the internal mammary artery, and peripheral and lateral 


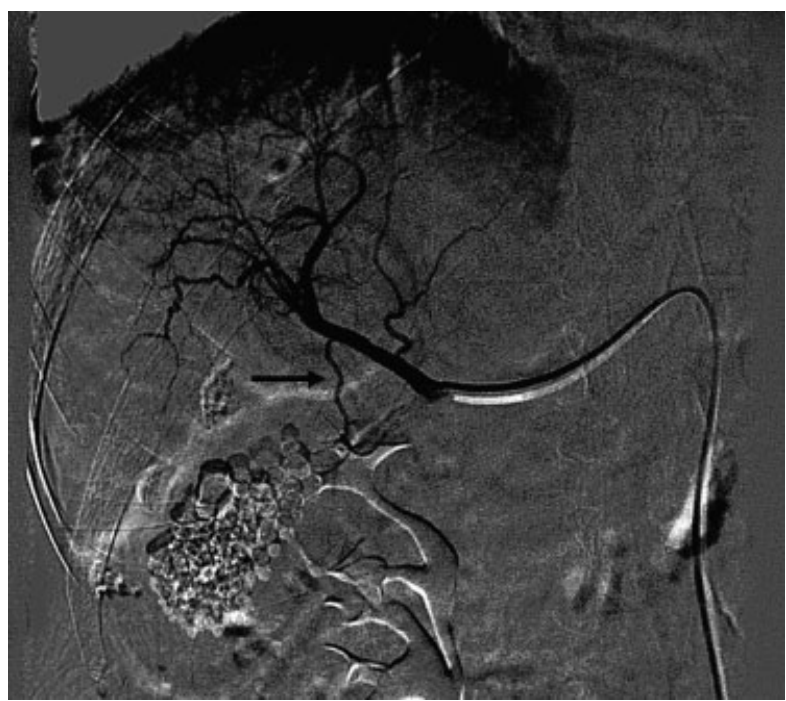

Figure 2 A 45-year-old man presents for transarterial chemoembolization (TACE). Right hepatic arteriogram shows prominent cystic artery (arrow). Although the cystic artery should be avoided during embolization, inadvertent embolization usually leads to self-resolving chemical cholecystitis.

tumors can parasitize intercostal or lumbar arteries. Care should be taken when embolizing these nonhepatic feeders as they can cause damage to the diaphragm or skin. In general, I use bland embolization (PVA only) for these vessels.

At our institution, we use a modified conventional TACE protocol using three chemotherapeutic agents in addition to drug-eluting beads. However, many practitioners use conventional TACE due to cost considerations, familiarity with procedure, and current lack of available evidence to suggest superiority of drug-eluting beads. Other practitioners use drug-eluting beads with doxorubicin only. I have listed below the preparation for all three possibilities.

\section{CHEMOTHERAPEUTIC MIXTURE PREPARATION}

1. Conventional TACE (triple agent or cisplatin, adriamycin, and mitomycin [CAM] protocol)

50 to $100 \mathrm{mg}$ doxorubicin (Pharmacia \& Upjohn, Kalamazoo, MI)

$50 \mathrm{mg}$ cisplatin powder (Bristol Myers Squibb, Princeton, $\mathrm{NJ})$

$10 \mathrm{mg}$ mitomycin (Bedford Laboratories, Bedford, $\mathrm{OH}$ )

These three drugs are reconstituted with a total of $10 \mathrm{~mL}$ of water soluble contrast material (Omnipaque 300; Winthrop Pharmaceuticals, New York, NY). Prior to intraarterial administration, emulsify in $10 \mathrm{cc}$ of iodized oil (Ethiodol; Savage Laboratories, Melville,
$\mathrm{NY}$ ) for a total of volume of $20 \mathrm{cc}$. Additional particle or gel foam embolization can be used at the end to achieve stasis in tumor vessels.

\section{Drug-eluting beads}

Two types of drug eluting beads are commercially available. They are used in conjunction with doxorubicin. For doxorubicin-loaded beads, select the tumor vessels and administer the drug-loaded beads. Larger tumors might require more embolic (more than one vial). I usually embolize the tumor vessels to stasis. In cases where stasis is not achieved with the drug-loaded beads, I use PVA particles to achieve stasis. With the triple CAM regimen, in addition to the doxorubicin-loaded beads, another syringe containing cisplatin and mitomycin is prepared and emulsified with iodized oil prior to intraarterial administration. Typically, the iodized oil preparation is used first until there is visible uptake of the oil and slowing of the flow in the feeder vessels. Then the doxorubicin loaded beads are infused until there is stasis. If there is no stasis after all the doxorubicinloaded beads are used, then PVA particles are used to achieve stasis.

3. Quadrasphere ${ }^{\circledR}$ microspheres, 100 to 150 microns (Biosphere Medical, Rockland, MA)

Single Agent

50 to $100 \mathrm{mg}$ of doxorubicin in 1 to 2 vials $\mathrm{LC}$ beads or Quadraspheres ${ }^{\mathbb{R}}$. Mix with $10 \mathrm{cc}$ of normal saline and set aside for at least one hour. When ready for intraarterial administration, decant and discard supernatant. Mix in equal amount of iso-osmolar nonionic contrast with bead solution.

Triple Agent (CAM)

Optional: $50 \mathrm{mg}$ cisplatin and $10 \mathrm{mg}$ mitomycin in $5 \mathrm{~mL}$ ethiodol

Syringe 1: Reconstitute $50 \mathrm{mg}$ doxorubicin with $10 \mathrm{cc}$ normal saline. Mix well to obtain clear solution. Add the reconstituted doxorubicin to 1 to 2 vials of Quadraspheres ${ }^{\mathbb{R}}$. Let sit for at least one hour. Draw out content and put into $10 \mathrm{cc}$ syringe. Prior to intraarterial administration, decant and discard supernatant $(\sim 5$ to $6 \mathrm{cc})$ and mix the remaining solution with $5 \mathrm{~mL}$ Visipaque ${ }^{\mathrm{TM}}$ (GE Healthcare, Chalfont St. Giles, UK).

Syringe 2: Prepare cisplatin and mitomycin: Add $5 \mathrm{cc}$ of normal saline to $10 \mathrm{mg}$ vial of mitomycin. Agitate to mix solution. Withdraw entire content and add to $50 \mathrm{mg}$ vial of cisplatin powder. Agitate to mix solution. Place the $5 \mathrm{cc}$ solution in a $10 \mathrm{cc}$ syringe. Prior to administration intraarterially, mix with $5 \mathrm{cc}$ ethiodol.

4. LC Beads, 300 to 500 microns (Angiodynamics, Queensbury, NY) 
Allow the vial of beads to stand and the beads will settle to the bottom. Aspirate as much of the saline as possible and discard. Reconstitute a vial of $50 \mathrm{mg}$ doxorubicin powder with $2 \mathrm{cc}$ sterile water and add to LC beads. The beads will turn from blue to red. Allow at least 30 minutes for the beads to adsorb the doxorubicin. Prior to use, get rid of as much supernatant as possible and add the same volume as the remaining bead solution of iso-osmolar nonionic contrast. For triple agent use, prepare another syringe with cisplatin and mitomycin as above.

\section{POSTPROCEDURE}

The use of antibiotics after the procedure is not routine at our institution, unless patients have a disrupted sphincter of Oddi. In these patients, antibiotics should cover Gram-negative enteric organisms. IV antibiotics could be converted to oral when patients are able to tolerate oral intake to facilitate discharge and should be continued for a complete 7 -day course. Antiemetics are used as indicated. For pain control, narcotics are used and we occasionally use patient controlled analgesia pump. Imaging follow-up is necessary to evaluate for response and plan for future therapy. Patients get cross-sectional imaging 6 to 8 weeks postprocedure. If there is objective response based on imaging but in the presence of viable tumor seen as contrast enhancement, the patient returns for repeat treatment. For patients without active disease, we follow with cross-sectional imaging every 3 to 4 months.

\section{DISCUSSION}

TACE has become accepted treatment due to the work of many investigators despite early and frequent skepticism by the medical community. Early studies showed unresectable $\mathrm{HCC}$ response rate to TACE was 60 to $80 \%$ measured by decreased tumor volume and decreased $\alpha$-fetoprotein levels. ${ }^{9,10}$ The future of TACE was in doubt when in 1995, a multicenter European trial showed no significant improvement in survival with TACE. However, this study excluded patients with more severe disease and used nonstandardized chemotherapeutic agents. ${ }^{11}$ Nonetheless, the fact that this study which was published in the New England Journal of Medicine was convincing enough for many to cast doubt on the procedure despite a separate study with contradictory results published contemporaneously in Cancer. This second study was a French multicenter trial consisting of a more representative sample of 127 patients, using standard agents, showing improved survival rate of TACE over the control group (64\% and $18 \%$ at one year and $38 \%$ and $6 \%$ at 2 years, respectively; $p<0.0001$ ) with improved quality of life overall. $^{12}$
HCC when small and in patients with good liver functions is best treated with resection. Liver transplantation can eliminate the tumor and also address the underlying disease. The use of TACE has been shown to have survival benefits in patients with unresectable HCC. Some authors advocate bland embolization, ${ }^{13}$ using permanent particles, there is no randomized trial showing effectiveness of bland embolization over chemoembolization. However, there is a phase 2 randomized single-blind controlled trial of Bead Block microspheres versus doxorubicin loaded LC beads (NCT00539643). The Barcelona randomized controlled trial published in 2002 actually showed superiority of TACE over bland embolization using Gelfoam ${ }^{\circledR} .{ }^{14}$ The trial was stopped prematurely when it became evident that chemoembolization conferred a significant survival benefit over conservative treatment. With increasing use of TACE, complications have been studied in details and the overall rate is in the range of 2.1 to $12 \%{ }^{8}$ More recently, with the advent of drug eluting beads, there has been hope of a more effective treatment for unresectable HCC. There are additional trials evaluating the effect of drugs such as sorafanib and chemoembolization (NCT00844883). There are many new possibilities of treating unresectable $\mathrm{HCC}$, the ultimate technique or combination of techniques remains to be determined.

\section{REFERENCES}

1. El-Serag HB. Hepatocellular carcinoma: an epidemiologic view. J Clin Gastroenterol 2002;35(5, Suppl 2):S72-S78

2. Fattovich G, Stroffolini T, Zagni I, Donato F. Hepatocellular carcinoma in cirrhosis: incidence and risk factors. Gastroenterology 2004;127(5, Suppl 1):S35-S50

3. Mazzaferro V, Regalia E, Doci R, et al. Liver transplantation for the treatment of small hepatocellular carcinomas in patients with cirrhosis. N Engl J Med 1996; 334(11):693-699

4. Yao FY, Ferrell L, Bass NM, Bacchetti P, Ascher NL, Roberts JP. Liver transplantation for hepatocellular carcinoma: comparison of the proposed UCSF criteria with the Milan criteria and the Pittsburgh modified TNM criteria. Liver Transpl 2002;8(9):765-774

5. Freeman RB Jr. Transplantation for hepatocellular carcinoma: The Milan criteria and beyond. Liver Transpl 2006; 12(11, Suppl 2):S8-S13

6. Yao FY. Selection criteria for liver transplantation in patients with hepatocellular carcinoma: beyond tumor size and number? Liver Transpl 2006;12(8):1189-1191

7. Lubienski A. Hepatocellular carcinoma: interventional bridging to liver transplantation. Transplantation 2005;80(1, Suppl): S113-S119

8. Kothary N, Weintraub JL, Susman J, Rundback JH. Transarterial chemoembolization for primary hepatocellular carcinoma in patients at high risk. J Vasc Interv Radiol 2007;18(12):1517-1526, quiz 1527

9. Nakamura H, Hashimoto T, Oi H, Sawada S. Transcatheter oily chemoembolization of hepatocellular carcinoma. Radiology 1989;170(3 Pt 1):783-786 
10. Venook AP, Stagg RJ, Lewis BJ, et al. Chemoembolization for hepatocellular carcinoma. J Clin Oncol 1990;8(6):1108-1114

11. A comparison of lipiodol chemoembolization and conservative treatment for unresectable hepatocellular carcinoma. Groupe d'Etude et de Traitement du Carcinome Hépatocellulaire. N Engl J Med 1995;332(19):1256-1261

12. Bronowicki JP, Vetter D, Dumas F, et al. Transcatheter oily chemoembolization for hepatocellular carcinoma. A 4-year study of 127 French patients. Cancer 1994;74(1):16-24
13. Maluccio MA, Covey AM, Porat LB, et al. Transcatheter arterial embolization with only particles for the treatment of unresectable hepatocellular carcinoma. J Vasc Interv Radiol 2008;19(6):862-869

14. Llovet JM, Real MI, Montaña X, et al; Barcelona Liver Cancer Group. Arterial embolisation or chemoembolisation versus symptomatic treatment in patients with unresectable hepatocellular carcinoma: a randomised controlled trial. Lancet 2002;359(9319):1734-1739 